\title{
Probabilistic Life Prediction for Crack Initiation on Coke Drums Based on Monte Carlo Simulation
}

\author{
Wei-Wen YE ${ }^{1}$, Wen-Feng WANG ${ }^{1, a}$, Zhi-Hua NING ${ }^{2, b^{*}}$, Ren-Huai LIU² and \\ Fan WANG ${ }^{2}$
} ${ }^{1}$ Guangzhou Special Pressure Equipment Inspection \& Research Institute, Guangzhou, 510663,
China

${ }^{2}$ College of Mechanics and Construction Engineering, Jinan University, Guangzhou, 510632, China

aemail: hpccpu@126.com, bemail: tningzhihua@jnu.edu.cn

Keywords: Coke drum, Crack initiation, Monte carlo simulation, Probabilistic life.

\begin{abstract}
High thermal stresses developed in coke drums can lead to fatigue crack initiation and subsequently growth until premature drum failure. To predict the fatigue crack initiation lives of coke drums, a cost-efficient method was proposed using Monte Carlo simulation in this paper. Strain data on the critical location of the drum wall was collected through the field measurement. Then statistical analysis was performed to build the probabilistic model of strain amplitude distribution. Large number of strain data could be generated by sampling on the basis of the probabilistic model. The fatigue crack initiation life was calculated according to Manson-Coffin equation and Palmgren-Miner's cumulative damage rule. The crack initiation was predicted probabilistically and the predicting result was in good agreement with the inspection history. The method developed in the present study is expected to be helpful for the future maintenance and inspection of coke drums.
\end{abstract}

\section{Introduction}

Coke drums are vertical pressure vessels that experience severe thermal cycling conditions in petroleum refineries. The consequential high thermal stresses, applied in a cyclic manner, can lead to premature drum failure in the form of through wall low cycle fatigue cracking. Therefore, it is important conduct reliable fatigue life assessment for coke drums.

Fatigue can be divided into stress-controlled fatigue and strain-controlled fatigue according to the applied stress level during cycles. If the maximum stress is less than the yield strength of the materials, it is called stress-controlled fatigue, in which the number of life cycles is at a high level. This kind of failure is also called high-cycle fatigue (HCF). If the maximum cyclic stress is greater than the yield strength, it is called strain-controlled fatigue. In strain-controlled fatigue, the cyclic stress is at a high level and the number of life cycles is usually less than $10^{4}$, namely low cycle fatigue (LCF).

The data from field measurement also showed that the peak value of the stress on the drum wall is higher than the material yield strength. Typical period of the cycles varies from 36 to 48 hours and total life of coke drums is always between 20 and 30 years. Many researchers found that low cycle fatigue is considered the failure mode for coke drums [1-3]. There are two methods widely used in fatigue life estimation, stress-based approach and strain-based approach. Strain has been shown to be an excellent quantity for correlating with low-cycle fatigue. For the purposes of the present analysis, strain-based approach is adopted on the fatigue crack initiation estimation. Lots of research work focused on the crack propagation and remaining life assessment, such as [4-5]. However, the crack initiation estimation is also important to the operators, which can help them to make a decision regarding the future maintenance and inspection strategy [6-8].

In this paper, a cost-efficient method for predicting the fatigue crack initiation life of coke drums has been presented using Monte Carlo simulation. Strain data on the critical location of the drum wall was collected through the field measurement, and then statistical analysis was performed to build the probabilistic model of strain amplitude distribution. Large number of strain data could be 
generated by sampling on the basis of the probabilistic model. Finally, the cumulative crack initiation probabilities were predicted for the future life in the coke drum service.

\section{Strain-Life Approach based on Manson-Coffin Equation}

The total strain verse fatigue life can be written as

$$
\varepsilon_{a}=\frac{\sigma_{f}^{\prime}}{E}(2 N)^{b}+\varepsilon_{f}^{\prime}(2 N)^{c}
$$

where $\varepsilon_{a}$ is the total strain amplitude, $\varepsilon_{a}=\Delta \varepsilon_{e} / 2+\Delta \varepsilon_{p} / 2, \Delta \varepsilon_{e}$ and $\Delta \varepsilon_{p}$ are the elastic and plastic strain amplitude respectively, $E$ is the Young's modulus, $\sigma_{f}^{\prime}$ is fatigue strength coefficient, and $\mathrm{b}$ is fatigue strength exponent with the range of $-0.06 \sim-0.14$. $\varepsilon_{f}^{\prime}$ is the fatigue ductility coefficient and $\mathrm{c}$ is the fatigue ductility exponent with the value between -0.7 and -0.5 . $2 N_{f}$ is reversals to failure.

In 1950s, Manson[9] and Coffin[10] found that the plastic strain is predominant under high temperature and low cycles fatigue condition, and the elastic strain can be neglected, i.e. $\varepsilon_{a} \approx \Delta \varepsilon_{p} / 2$. They independently proposed an identical expression to correlated the strain and fatigue life as follows

$$
\frac{\Delta \varepsilon_{p}}{2}=\varepsilon_{f}^{\prime}\left(2 N_{f}\right)^{c}
$$

Thus, the crack initiation life $N_{f}$ can be expressed by

$$
N_{f}=\frac{1}{2}\left(\frac{\Delta \varepsilon_{p}}{2 \varepsilon_{f}^{\prime}}\right)^{\frac{1}{c}}
$$

In 1960s, it was found in two-dimensional tension-torsion and bending-torsion fatigue test that the result of fatigue life assessment using the equivalent strain is the same as that of using uniaxial tension.

The coke drum wall can be treated as an axial symmetric component, in which the maximum equivalent strain amplitude $\Delta \varepsilon_{e q} / 2$ based on Von Mises criterion can be calculated by [11]

$$
\frac{\Delta \varepsilon_{e q}}{2}=\frac{2}{\sqrt{3}} \sqrt{\left(\frac{\Delta \varepsilon_{z}}{2}\right)^{2}+\left(\frac{\Delta \varepsilon_{\theta}}{2}\right)^{2}+\frac{\Delta \varepsilon_{\theta}}{2} \frac{\Delta \varepsilon_{z}}{2}}
$$

where $\Delta \varepsilon_{z} / 2$ and $\Delta \varepsilon_{\theta} / 2$ are the axial and hoop strain amplitudes that produce the maximum equivalent strain amplitude, respectively.

\section{Crack Initiation Analysis Using Monte Carlo Simulation}

Although the delayed coking process keeps unchanged, the field measurement shows that the strain or stress on the drum wall varies from cycle to cycle. This may be led by multiply factors, such as fluctuant internal pressure or temperature. But the most dominant reason should be as follows: In the quench stage some cold water maybe flow through the channels in the coke bed and quench the drum wall directly, which will cause severe thermal gradient. In general, locations of the channel in the coke bed are random. So the cycle is not a constant-amplitude one. On-line monitoring with high temperature strain gauges installed at the critical locations on the drum is a common way which can provide true cyclic strain amplitude. However, the cost would be very high to perform long-term monitoring. 
A cost-efficient way based on Monte Carlo simulation could be developed in this paper. First, strain amplitude at a critical location is monitored and collected over a number of cycles. Then statistical analysis will be performed on the collected data, and the probabilistic distribution model can be built for the strain at the selected location. According to this probabilistic distribution model, Monte Carlo simulation is applied and strain data of large sample size would be generated. Finally, probabilistic life assessment of crack initiation could be inferred based on these data. The results can be used to quantify the likely risks associated with future operation and making decisions regarding future maintenance and inspection strategies.

The fatigue test shows that there is little variability in the fatigue ductility coefficient and fatigue ductility exponent, i.e. $\varepsilon_{f}^{\prime}$ and c could be considered as certainty valuables. The strain amplitude has a log-normal distribution under usual working condition. It can be inferred from Eq.(3) that the crack initiation life $N_{f}$ has a log-normal distribution as well.

If the probability of crack initiation is $p$, the associated crack initiation life $N_{f}^{p}$ can be written as

$$
N_{f}^{p}=\lg ^{-1} x_{p}
$$

where $x_{\mathrm{p}}$ represents the logarithmic life with the probability $p$, which has a normal distribution.

$$
\begin{aligned}
& x_{p}=\bar{x}+u_{p} S, \bar{x}=\frac{1}{n} \sum_{i=1}^{n} x_{i}, \\
& S=\sqrt{\frac{1}{n-1} \sum_{i=1}^{n}\left(x_{i}-\bar{x}\right)^{2}}
\end{aligned}
$$

where $\bar{x}$ and $S$ are the sample mean and standard deviation corresponding to sample size $n$, respectively. $u_{\mathrm{p}}$ is the quantile of order $p$ for standard normal distribution.

For a certain confidence level $1-\alpha$, the logarithmic life is given by

$$
x_{p, 1-\alpha}=\bar{x}+k S
$$

where

$$
k=\frac{u_{p}-u_{1-\alpha} \sqrt{\frac{1}{n}\left[1-\frac{u_{1-\alpha}^{2}}{2(n-1)}\right]+\frac{u_{p}^{2}}{2(n-1)}}}{1-\frac{u_{1-\alpha}^{2}}{2(n-1)}}
$$

and $u_{1-} \quad$ satisfies the following relationship

$$
P\left\{\frac{x_{p}-\bar{x}_{p}}{S} \leq u_{1-\alpha}\right\}=1-\alpha
$$

\section{Application on a Coke Drum}

The base shell of the monitored coke drum was made of low alloy steel $15 \mathrm{CrMoR}$. The cycle period is $36 \mathrm{~h}$.

To obtain fatigue coefficients of $15 \mathrm{CrMoR}$, tensile fatigue tests were carried out on MTS- 810 type machine. The design of the test specimens, test procedure and data analysis were all performed according to GB/T15248 - 94 . 
Table 1. Summary of LCF test results

\begin{tabular}{|c|c|}
\hline$\Delta \varepsilon_{\mathrm{p}} / 2$ & $2 N_{f}$ \\
\hline 0.0021769 & 9594 \\
\hline 0.0040256 & 3026 \\
\hline 0.0058114 & 1346 \\
\hline 0.0077307 & 692 \\
\hline 0.0097409 & 540 \\
\hline 0.012564 & 384 \\
\hline
\end{tabular}

The whole tests were divided into six groups, associated to different strain level: $0.4 \%, 0.6 \%$, $0.8 \%, 1.0 \%, 1.2 \%$ and $1.5 \%$. There were three specimens in every group. The load spectrum was in the form of triangle wave, with strain ratio $R_{\varepsilon}=-1$. The test results were shown in Table 1 .

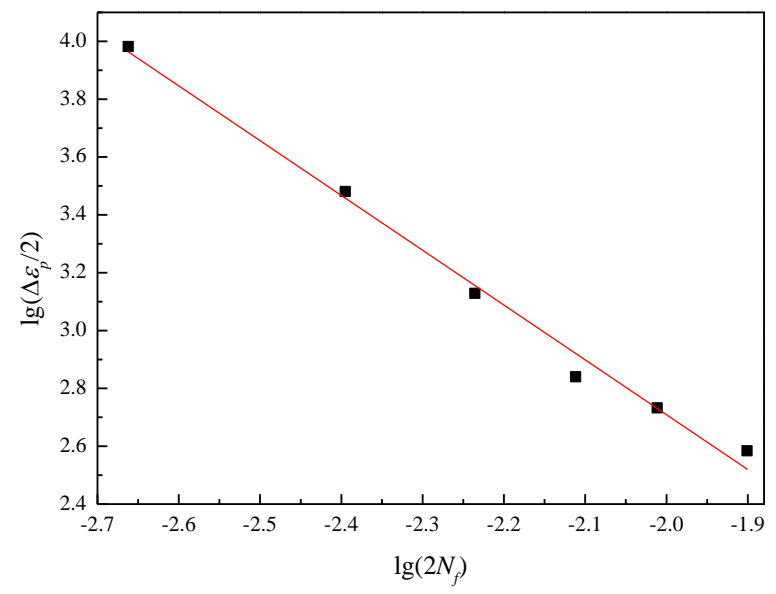

Figure 1. Plastic strain amplitude versus $2 N_{f}$

Since the failure of coke drums is LCF, the strain-life relationship in Eq.(2) was used in the experiment data analysis. It was shown in Fig.1 that a linear function between plastic strain amplitude and reversal life in log-log scale.

The Manson-coffin equation can be obtained through least squares fitting as

$$
\frac{\Delta \varepsilon_{p}}{2}=0.2690\left(2 N_{f}\right)^{-0.5278}
$$

The monitored location was selected on the shell close to the circumferential weld seam at the lower part of the coke drum. This is a typical critical location, where thermal strains are always at a high level to lead to crack initiation. The monitoring process lasted about six months covering totally 108 cycles. The equivalent strain amplitudes were calculated according to Eq.(4). After applying logarithmic operation and statistical analysis, the histogram of the equivalent strain amplitudes was shown in Fig.2. It can be seen that the equivalent strain amplitude could be considered having a log-normal distribution with the mean value -2.3675 and standard deviation 0.2306 , i.e. $\lg \frac{\Delta \varepsilon_{e q}}{2} \sim N\left(-2.3675,0.2306^{2}\right)$.

According to Palmgren-Miner's cumulative damage model, the fatigue cumulative damage is calculated as [12] 


$$
D=\sum_{i=1}^{n} \frac{n_{i}}{N_{f i}}
$$

where $n_{i}$ is the number of cycles at a given strain amplitude, and $N_{f i}$ is the number of cycles to crack initiation at the same strain amplitude. $D$ is the damage index and the damage at crack initiation is taken as $D=1$.

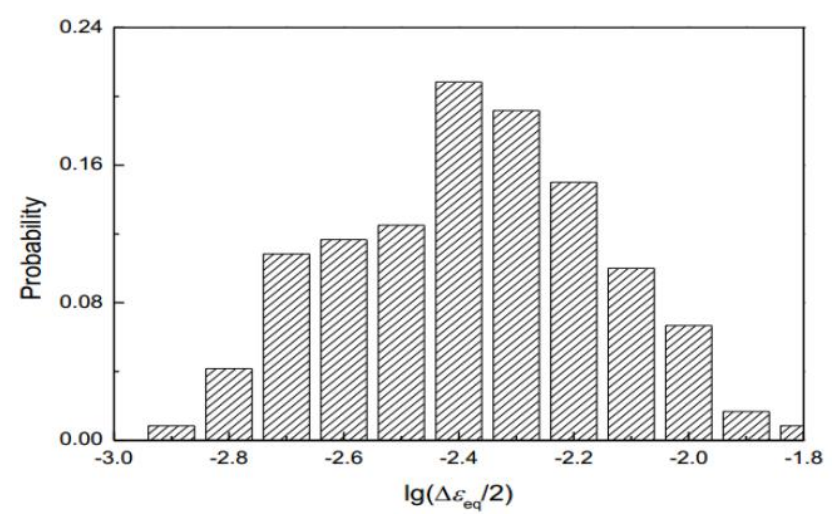

Figure 2. Histogram of equivalent strain amplitude

In the present work, the crack initiation estimation is performed probabilistically.

The procedure of the corresponding algorithm is as follows:

i. Input material properties;

ii. According the distribution the equivalent strain amplitude, perform sampling to the equivalent strain amplitude, and then calculate the associated $N_{f}$ and fatigue damage.

iii. Apply Miner' rule to accumulate the total fatigue damage under a given number of cycles. For a given number of cycles, the associated crack initiation probability is calculated based on a large sample.

iv. The confidence interval of the designated confidence level is calculated for every given number of cycles.

The cumulative probability of fatigue crack initiation was shown in Fig.3, in which the sample capacity is taken as 20000, the confidence level $1-\alpha=95 \%$. It was shown in Fig. 3 that when the operational cycles exceed 1900(about 8 years), the probability of crack initiation increased to 0.5 . Actually, cracking has indeed occurred in the past and was found in this location in the inspection seven months ago, about 8.5 years (2040 cycles) since commissioning. The predicted response aligned well with the inspection finding.

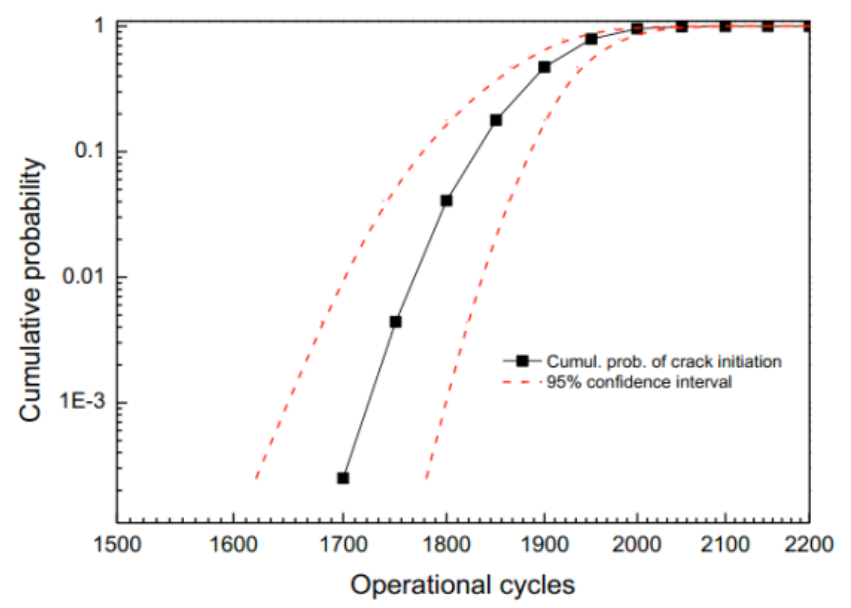

Figure 3. Cumulative probability of crack initiation 


\section{Conclusion}

A cost-efficient method for assessing the fatigue crack initiation in coke drums has been presented using Monte Carlo simulation in the present study. The crack initiation life predicted in probabilistic form was more credible for the operators of coke drums. It can help them to make a decision regarding future maintenance and inspection strategy on the level risk.

With respect to the next work in the future, it should be noted that strain data from larger number of cycles would help to build a more accurate probabilistic model. Moreover, coke drums actually experience complex thermal-mechanical loading condition, thus fatigue properties from thermal-mechanical cyclic test should be used in Manson-coffin equation. Chen and Xia [6] found that the heat affected zone (HAZ) has the shorter period for crack initiation. The result could be used to explain why a lot of cases observed that cracks were near or within the HAZ. This should be also considered in the future study, which would help to get more reliable evaluation of crack initiation.

\section{Acknowledgement}

This work was supported by the National Natural Science Foundation of China (No.11302083) and funded by Guangzhou Special Pressure Equipment Inspection \& Research Institute.

\section{References}

1. J.A. Penso, Y.M. Lattarulo, A.J. Seijas, J. Torres, D. Howden, C.L. Tsai. Press Ves Pip, ASME 395, 243-253(1999)

2. C.J. Pieper, L.R. Shockley, C.W. Stewart. AIChE 2000 Spring National Meeting (Atlanta, GA, March 5-9, 2000)

3. Boswell R. Coke drum bulges (Stress Eng Services, 2001)

4. H. Ambarita, M. Daimaruya, H. Fujiki, H. Kawai, H. Kobayashi, M. Oka. J Therm Stresses 35, 1095-1107(2012)

5. Boswell R S, Ferraro T. Plan engineering, design and responsibility symposium, energy engineering conference (Remaining life evaluation of coke drums, 1997)

6. J. Chen, Z. Xia. Mater Design 63, 575-583(2014)

7. J.M. Church, L.B. Lim, J.M. Brear, P. Jarvis, RPD Lant, C.J. Middleton. Int J Pressure Vessels Piping 78, 1011-1020(2001)

8. Z. Yan, Y. Zhang, J. Chen, Z. Xia, Y. Zhang. Eng Fail Anal 48, 259-271(2015)

9. S.S. Manson. Heat transfer Symposium,9-75(1953)

10. L. F. Coffin. Trans ASME 76, 931-950 (1954).

11. ASME Boiler and Pressure Vessel Code (Part 5, section VIII, rules for construction of pressure vessels, division 2, 2010)

12. M.A. Miner. J Appl Mech Trans ASME 12, A159-64(1945) 